\title{
Wavelength Assignment Algorithm for Optical Networks
}

Gulzar Ahmad Dar ${ }^{1}$,Ifrah $\mathrm{Amin}^{2}$, and Hardeep Singh Saini ${ }^{3}$

1. Department of ECE, Indo Global College of Engineering, Mohali, Punjab, India faisalgulzar11@gmail.com

2. Department of ECE, Indo Global College of Engineering, Mohali, Punjab, India Ifrah.amin19@gmail.com

3. Professor in Department of ECE, Indo Global College of Engineeriing, Mohali, Punjab, India hardeep_saini17@yahoo.co.in

\section{ABSTRACT}

Wavelength assignment problem is one of the important problem in optical networks as on the first stage the route of the optical network is to be selected and after the route is selected then the wavelength is to be assigned to that route. In this paper we have proposed a wavelength assignment technique for the better performance of the optical network. The results have proved it better than the conventional algorithms.

\section{Council for Innovative Research}

Peer Review Research Publishing System

Journal: INTERNATIONAL JOURNAL OF COMPUTERS \& TECHNOLOGY

Vol 9, No 2

editor@cirworld.com

www.cirworld.com, member.cirworld.com 


\subsection{Introduction}

The rapid growth of Internet traffic has been the driving force for faster and more reliable data communication networks. Networking is a very promising technology to meet these ever increasing demands. Broadly speaking a computer network is an interconnected collection of interdependent computers that aid communication in numerous ways. Apart from providing a good communication medium, cost effectiveness and sharing of available resources (programs and data) are some of the advantages of networking. This rapid growth of internet traffic has been the driving force for faster and more reliable computer and data communication networks. Wavelength division multiplexing (WDM) is a very promising technology to meet the ever increasing demands of high capacity and bandwidth. In a WDM network several optical signals are sent on the same fiber using different wavelength channels. Sometimes, the term dense wavelength division multiplexing (DWDM) is used to distinguish the technology from the broadband WDM systems where two widely separated signals (typically $1310 \mathrm{~nm}$ and 1550nm) share a common fiber. In DWDM up to 40 or 80 signals are combined on the same fiber. WDM networks are a viable solution for emerging applications, such as supercomputer visualization and medical imaging, which need to provide high data transmission rate, low error rate and minimal propagation delay to a large number of users [1] Traditionally, only a small fraction of the fiber capacity was used, but by using WDM it is possible to exploit this huge capacity more efficiently [2]. The possibility to use the existing fibers more efficiently makes WDM a very attractive alternative commercially, as it is very expensive to install new fibers in the ground. This is the case especially in densely populated areas like cities, where fibers must be dug under streets etc. WDM technology has been recognized as one of the key components of the future networks. The commercialization of WDM technology is progressing rapidly. Most important for the development of the WDM technology was the invention of Erbium Doped Fiber Amplifier, (EDFA) an optical fiber amplifier in 1987. The optical fiber amplifier is a component capable of amplifying several optical signals at the same time without converting them first to electrical domain (opto-electronic amplification). It is also important to note that EDFAs can be used to amplify signals of different bit rates and modulations. Other important WDM components include lasers, receivers, wavelength division multiplexers, wavelength converters, optical splitters and tunable filters amongst others. There is also wide interest towards the optical networking in academic community as it offers a rich research field for scientists from the component level up to the network protocols.

\subsection{Wavelength Division Multiplexing}

Theoretically, fiber has extremely high bandwidth (about $25 \mathrm{THz}$ [terahertz]) in the 1.55 low-attenuation band and this is thousands times of the total bandwidth of radio on the planet Earth [3]. However, only speed of a few gigabits per second is achieved because the rate at which an end user (a workstation) can access a network is limited by electronic speed, which is a few gigabits per second. Hence, it is extremely difficult to exploit all the bandwidth of a single fiber using a single high-capacity wavelength channel due to optical-electronic bandwidth mismatch or "electronic bottleneck." The recent breakthroughs (Tb/s) are the result of two major developments: WDM, which is a method of sending many light beams of different wavelengths simultaneously down the core of an optical fiber and the EDFA, which amplifies signal at different wavelengths simultaneously regardless of their modulation scheme or speed. WDM is essentially same as frequency division multiplexing (FDM), which has been used in radio systems for more than a century. WDM systems use a carrier wave which is higher than that of an FDM channel by a million times in frequency (THz versus $\mathrm{MHz}$ ). Within each WDM channel, it is possible to have FDM where the channel bandwidth is subdivided into many radio frequency channels each at a different frequency. This is called subcarrier multiplexing. A wavelength can also be shared among many nodes in a network by electronic time division multiplexing. Note that WDM eliminates the electronic bottleneck by dividing the optical transmission spectrum (1.55-micron band) into a number of non-overlapping wavelength channels. These channels coexist on a single fiber with each wavelength supporting a single communication channel operating at a peak electronic speed. The attraction of WDM is that a huge increase in available bandwidth can be obtained without the huge investment necessary to deploy additional optical fiber. The DWDM technique effectively increases the total number of channels in a fiber by using very narrow spaced channels [54]. Typically channel spacing ranges from 0.4 $\mathrm{nm}$ to $4 \mathrm{~nm}$.

\subsection{WDM Optical Network}

WDM Optical Network is a network of computers in which the backbone is optical fiber cable and the mode of transmission is wavelength division multiplexing. The information stream from multiple sources is optically combined by the star and the signal power of each stream is split and forwarded to all nodes through their fibers. Communication between source and destination may either be singlehop or it may be multi-hop.

\section{Literature Review}

Z. Zhang et. al. [3], presented a heuristic algorithm for effective assignment of a limited number of wavelengths among the access stations of a multi-hop network where the physical medium consists of optical fiber segments which interconnect wavelength elective optical switches.

PoompatSaengudomlertet. al. [4],developed an on-line wavelength assignment algorithm for a wavelength-routed WDM tree network. The algorithm dynamically supports all k-port traffic matrices among end nodes. Implementation of proposed wavelength assignment algorithm was also demonstrated using a hybrid wavelength-routed/broadcast tree with only one switching node connecting several passive broadcast sub-trees.

Junjun Wan et. al. [5], proposed a wavelength assignment algorithm, which was based on the method called Dynamic Preferred Wavelength Sets (D-PWS). Also, they described the basic architecture of the optical burst switching network based on Dynamic Wavelength Routing (DWR), under which the guarantee of the quality of service in the DWR-OBS network was discussed. Then they focused on two aspects: the transmission latency of the data packets and the blocking probability, which leads to a quantitative description of the transmission latency and the size of the edge node buffer.

F. Matera et. al. [6], showed how to obtain a wavelength assignment in a wide geographical transport network connecting the main cities of Europe, when all optical wavelength converters are introduced in the network nodes. They also reported an investigation on $40 \mathrm{~Gb} / \mathrm{s}$ transmission performance in the presence of all optical wavelength converters based on four wave mixing in semiconductor optical amplifiers and on different frequency generation in periodically poled lithium niobate waveguides.

Anwar Alyatama [7], used random and first-fit wavelength assignment approach for presenting an approximate analytical method and evaluated the blocking probabilities in wavelength division multiplexing networks without wavelength converters. The new approach viewed the WDM network as a set of different layers (colours) in which, blocked traffic in one layer is overflowed to another 
layer. Analysing blocking probabilities in each layer of the network is derived from an exact approach. A moment matching method was then used to characterise the overflow traffic from one layer to another.

Raja Dattaet. al. [8], presented a wavelength assignment algorithm which was used for optimal assignment of a single wavelength to single-hop traffic in a tree topology. The work was further extended for the wavelength assignment in a general graph. This polynomial time algorithm gave an optimal solution to the routing and wavelength assignment problem in a tree topology.

P. Rajalakshmiet. al. [9], proposed a new wavelength assignment technique called wavelength reassignment algorithm in which when the new call gets blocked due to wavelength continuity constraint the already established calls were reassigned the wavelength, so as to create a wavelength-continuous route in order to accommodate the new call. During wavelength reassignment the routes for all calls remain the same, i.e. no rerouting was done. The problem of enhancing the blocking performance, in the circuit-switched wide-area optical wavelength-division multiplexed networks with no wavelength conversion at the nodes was also considered.

I. Alfouzanet. al. [10], introduced two new wavelength assignment reconfiguration algorithms, the One-Directional Transfer (1DT) and the Two-Directional Transfer (2DT) algorithms. The simulation results for both these algorithms were shown to outperform the existing algorithms in terms of the trade-off.

Abhisek Mukherjee et. al. [11], proposed a new wavelength conversion algorithm in a DWDM network using online routing. The model for the algorithm has been theoretically developed and the corresponding call connection probability has been calculated. The limitation on the number of wavelength conversions has been addressed by fixing the maximum number of wavelength conversions allowed for the transmissions of a single packet over the network.

Nen-Fu Huang et. al. [12], proposed an efficient distributed Wavelength Reusing/ Migrating /Sharing Protocol (WRMSP) for the Dual Bus Lightwave Networks (DBLN). This protocol constituted of three efficient schemes for carrying out the wavelength reusing, migration and sharing respectively.

Mahesh Sivakumaraet. al. [13], studied the effect of wavelength conversion on the blocking performance of connections with multiple rates. The blocking performance of the TDM wavelength routing network was evaluated through simulations.

\section{Proposed Algorithm}

In this section we have proposed an improvement of least used wavelength assignment algorithm. In this algorithm least-used wavelength assignment algorithm is executed until blocking. When the call is blocked wavelength conversion is introduced and hence blocking probability is reduced. If the full wavelength conversion is used after least-used wavelength assignment algorithm the blocking probability is reduced to a very large extent and its value reduces to a minimum possible value. As full wavelength conversion is costlier than sparse wavelength conversion so the sparse wavelength conversion is employed in this proposed algorithm. The least-used wavelength conversion algorithm can be easily explained with the following steps.

\section{Algorithm}

1. Initialize the number of channels

2. Initialize the total number of links

3. Initialize the value of load in Erlangs

4. Initialize the number of wavelengths used

5. Select the source destination pairs

6. Fix the value of total number of generations

7. For different values of links

a. Reserve memory for vectors of connections for every link

b. For different values of call generations

i. Calculate the shortest path between the source-destination

ii. Calculate the exponential distribution of the time until the next call arrives (according to Poisson arrival)

iii. Calculate the exponential distribution of the holding time

iv. Check the blocking for all connection vectors

v. If all connection are busy then there is blocking

vi. Else assign new channel to the connection (wavelength conversion takes place)

vii. Decrement all holding time

viii. Remove all free channels

8. Update the number of blocked calls

9. Clear all temp storage

10. Calculate the blocking probability in terms of \%age

11. Plot the values

\section{Results and Discussion}

In this section, the simulation results of proposed improved wavelength conversion algorithm have been shown. Also, the blocking probability of proposed algorithm is compared with the conventional algorithms. The simulation is carried out on simulation 
software MATLAB 7.2 of Mathworks. The blocking probability of network is compared depending upon number of channels, load and the number of links. The Improved wavelength conversion algorithm has been proposed for wavelength assignment and the performance of this wavelength assignment algorithmis evaluated in terms of blocking probability and fairness. The results are shown in figure $1-9$. In the first phase we have varied the number of wavelengths by keeping the other parameters constant. We have fixed the number of channels to 20; total number of links in the network to 20 and maximum load per unit link to 10 Erlangs and increased the number of wavelengths used from 20 to 50 respectively in figure 1 to 4 .

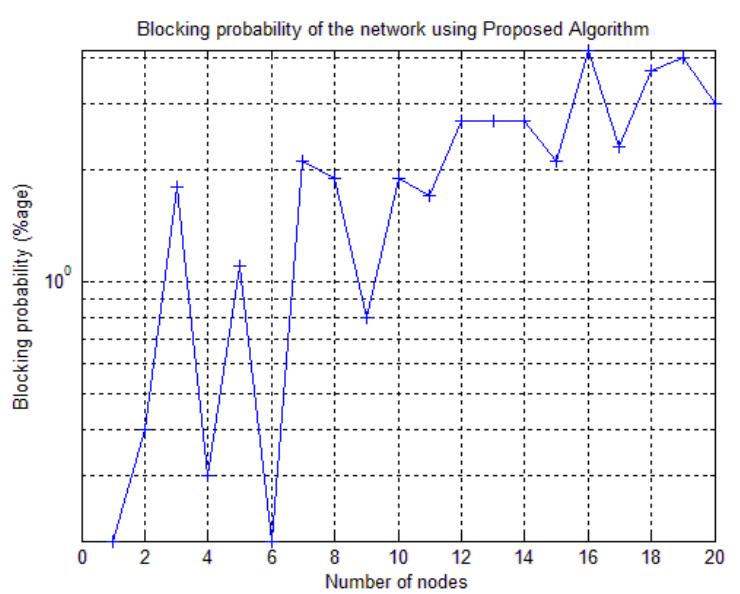

Figure 1: Blocking probability of the proposed Algorithms for $\mathrm{W}=10$.

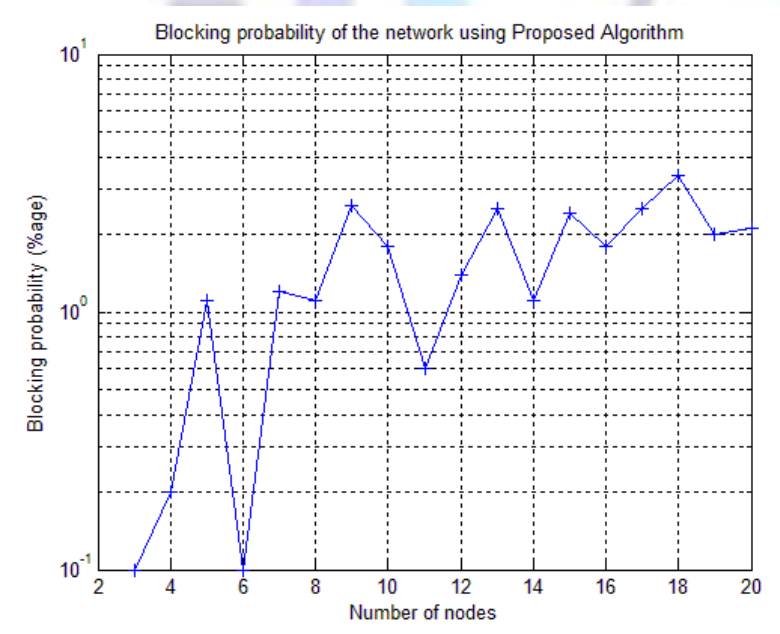

Figure 3: Blocking probability of the proposed Algorithms for $W=30$.

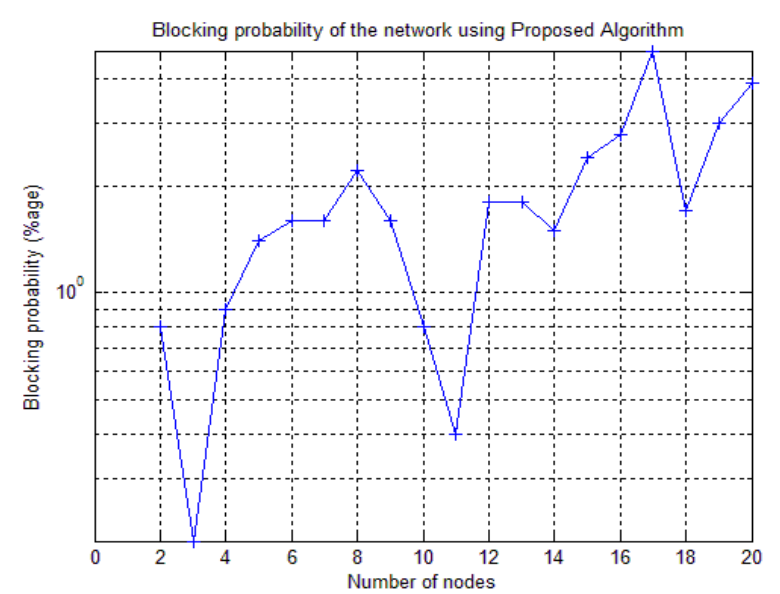

Figure 2: Blocking probability of the proposed Algorithms for $W=20$.

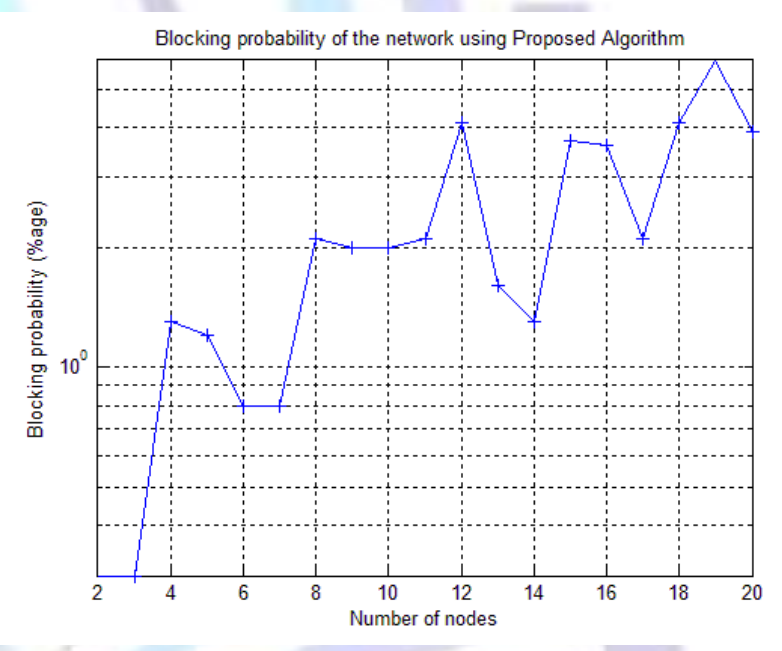

Figure 4: Blocking probability of the proposed Algorithms for $W=40$.

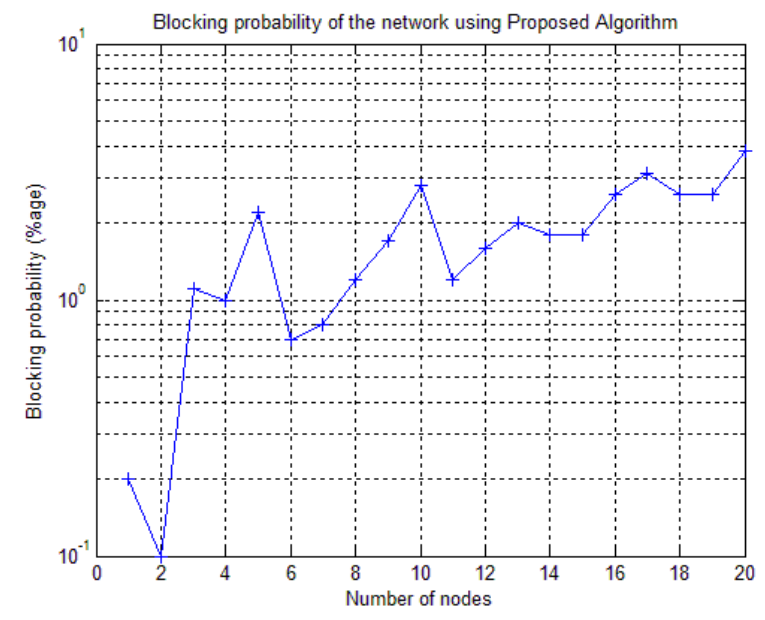

Figure 5: Blocking probability of the proposed Algorithms for $\mathrm{W}=50$. 
The results shown in figure $1-5$ prove that the blocking probability of the proposed algorithm decreases with the increase in the number of wavelengths. As the number of wavelength is increased the blocking probability is decreased. Further, in figure $6-9$ the load per unit link is increased keeping the other parameters constant. The results show that as the load is increased the blocking probability of the network increases for the proposed algorithm keeping other parameters constant.

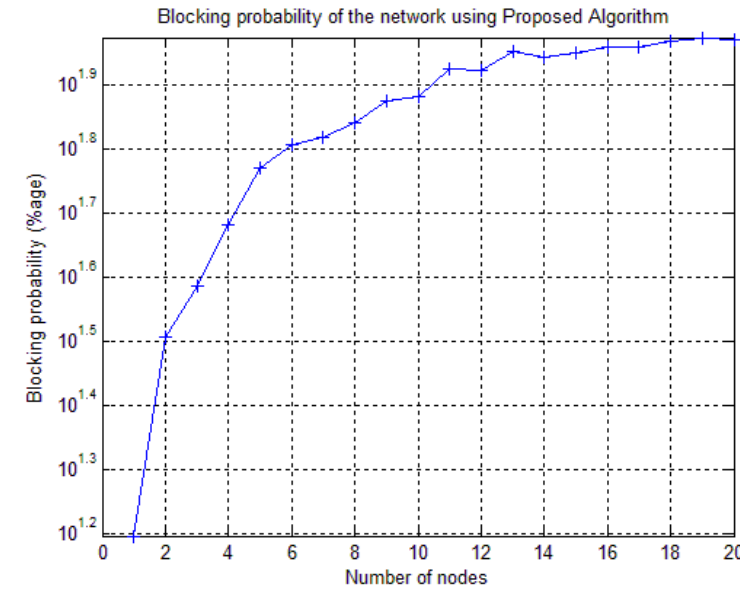

Figure 6: Blocking probability of the proposed Algorithms for load=20 Erl.

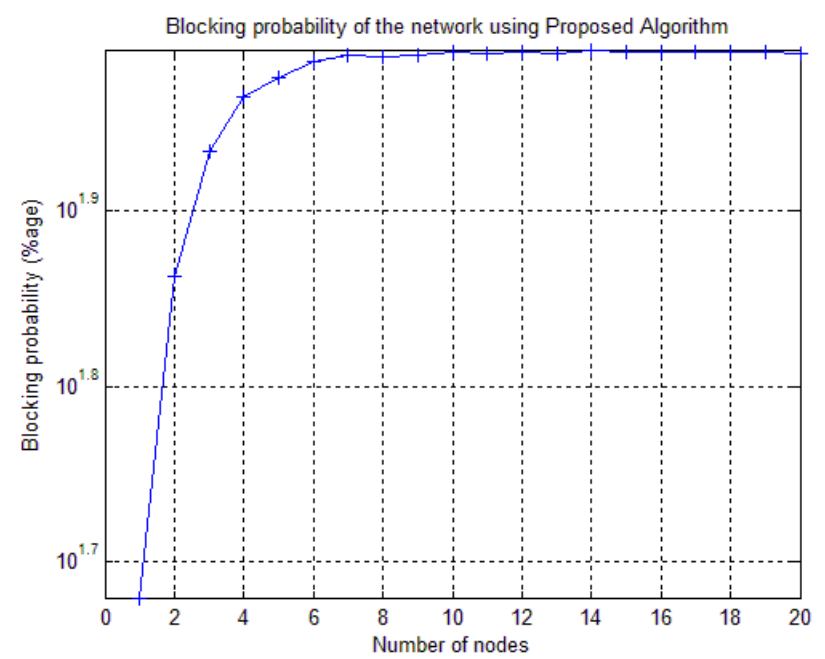

Figure 8: Blocking probability of the proposed Algorithms for

load $=40 \mathrm{Erl}$.

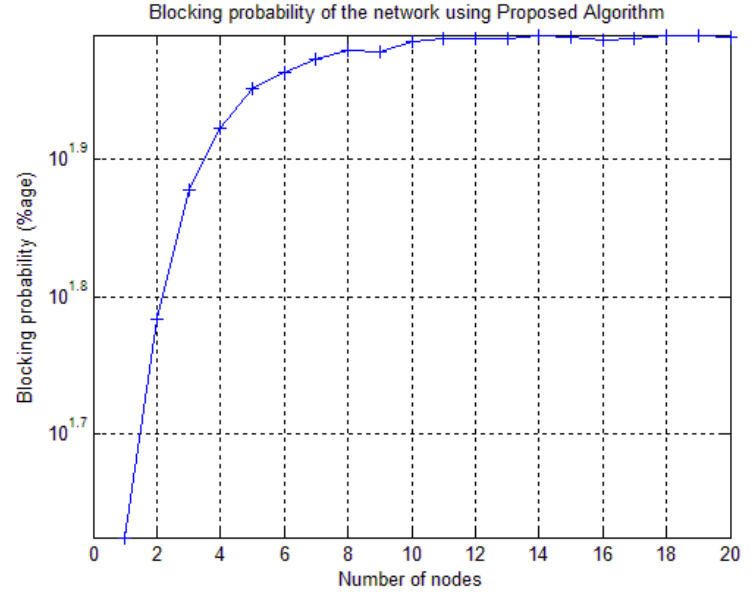

Figure 7: Blocking probability of the proposed Algorithms forload=30 Erl.

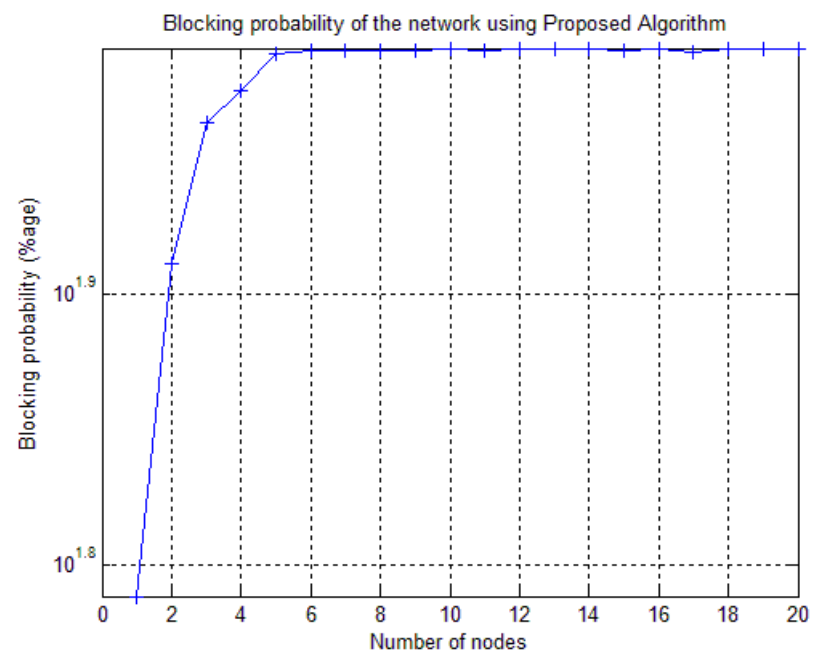

Figure 9: Blocking probability of the proposed Algorithms for load=40 Erl.

The results shown in figure 6-9 shows that as the load in increased the blocking probability of the network is increased.

\section{Conclusion}

This paper deals with the wavelength assignment algorithm and in this paper we have proposed an improved wavelength conversion algorithm. The performance of this algorithm is computed on the basis of blocking probability and fairness. The results show that the proposed algorithm is better in terms of blocking performance. In future this algorithm can be compared with the conventional algorithms.

\section{References}

[1]. R. Ramaswami, "Multi wave length lightwave networks for computer communication," IEEE Communication Magazine, Vol. 31, pp. 78-88, Feb. 1993.

[2]. AmitWason, R. S. Kaler, "Blocking in wavelength-routed all-optical WDM networks," Optik- International Journal for Light and Electron Optics- Elsevier Science, vol. 121, no. 10, pp. 903-907, June 2010.

[3]. Z. Zhang and A. S. Acampora, "A Heuristic Wavelength Assignment Algorithm for Multi-hop WDM Networks with Wavelength Routing and Wavelength Re-use," IEEE/ACM Transactions on Networking, vol. 3, no. 3, pp. 281-288, June1995.

[4]. PoompatSaengudomlert, Eytan H. Modiano and Robert G. Gallager, "Dynamic Wavelength Assignment for WDM All-Optical Tree Networks," IEEE/ACM Transactions on Networking, Vol. 13, No. 4, pp. 895-905, August 2005.

[5]. Junjun Wan, Yaling Zhou, Xiaohan Sun, Mingde Zhang, " Guaranteeing quality of service in optical burst switching networks based on dynamic wavelength routing," Optics Communications, vol. 220, pp. 85-95, 2003.

[6]. F. Matera, D. Forin, F. Matteotti, G. Tosi-Beleffi, "Numerical investigation of wide geographical transport networks based on 40 $\mathrm{Gb} / \mathrm{s}$ transmission with all optical wavelength conversion," Optics Communications, vol. 247, pp. 341-351, 2005. 
[7]. Anwar Alyatama, "Wavelength decomposition approach for computing blocking probabilities in WDM optical networks without wavelength conversions," Computer Networks, vol. 49, pp. 727-742, 2005.

[8]. Raja Datta, BivasMitra, SujoyGhose, and IndranilSengupta, "An Algorithm for Optimal Assignment of a Wavelength in a Tree Topology and its Application in WDM Networks," IEEE Journal on Selected Areas in Communications, vol. 22, no. 9, pp. 15891600, November 2004.

[9]. P. Rajalakshmi, Ashok Jhunjhunwala, "Wavelength reassignment algorithms for all-optical WDM backbone networks," Optical Switching and Networking, vol. 4, pp. 147-156, 2007.

[10]. I. Alfouzan, M.E. Woodward, "Some new load balancing algorithms for single-hop WDM networks," Optical Switching and Networking, vol. 3, pp. 143-157, 2006.

[11]. Abhisek Mukherjee, Satinder Pal Singh, V.K. Chaubey, "Wavelength conversion algorithm in an intelligent WDM network," Optics Communications, vol. 230, pp. 59-65, 2004.

[12]. Nen-Fu Huang and Shiann-TsongSheu, "An Efficient Wavelength Reusing/Migrating/Sharing Protocol for Dual Bus Lightwave Networks," Journal of Lightwave Technology, vol. 15, no. 1, pp. 62-75, January 1997.

[13]. Mahesh Sivakumara, Suresh Subramaniam, "Blocking performance of time switching in TDM wavelength routing networks," Optical Switching and Networking, vol. 2, pp. 100-112, 2005.
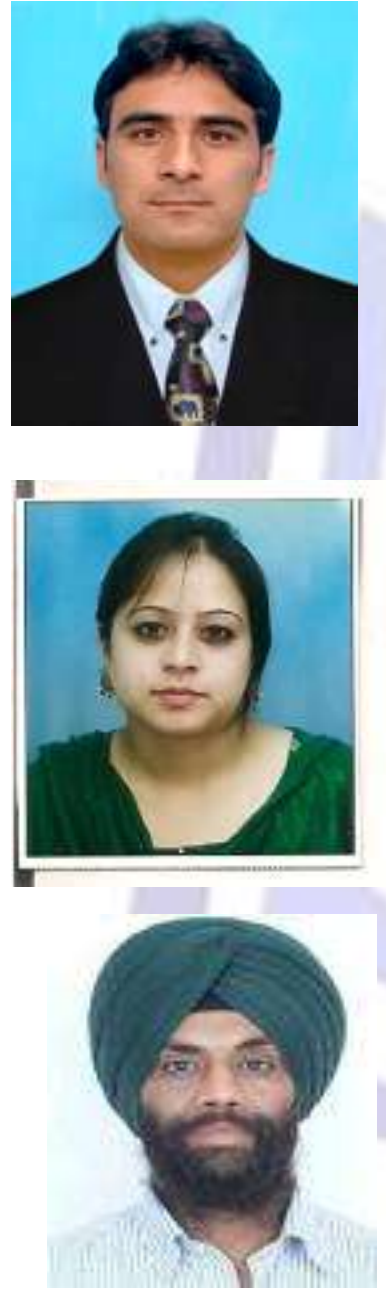

Gulzar Ahmad Dar completed his Engineering Diploma from State Board of Technical Education (Kashmir) and received his B Tech from Punjab Technical University Jalandhar and is Pursuing his M.Tech from Indoglobal College of Engineering Mohali India. His Research Interests include Optical Fiber Communication and Wave Length Assignments in Optical Networks.

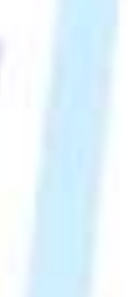

Ifrah Amin received his B Tech from Islamic University of Science and Technology Awantipora Kashmir and is pursuing M. Tech from Indo global College of Engineering Mohali, India. Her research interests in Optical Networks.

Hardeep Singh Saini obtained his Doctorate degree in Electronics \& Communication Engineering in 2012. He holds Master's degree in Electronics \& Communication Engineering from Punjab Technical University, Jalandhar passed in 2007. His total experience is 14 years, presently working as Professor(ECE) and Associate Dean Academic at Indo Global College of Engineering, Abhipur(Mohali), and PUNJAB (INDIA) since June-2007. He is author of 5 books in the field of Communication Engineering. He has presented 22 papers in international/national conferences and published 26 papers in international journals. He is a life member of the IETE (India), member of international association of engineers and member of international association of computer science and information technology (IACSIT).He is also a member of editorial/reviewer board of various international journals. 